\title{
Observational Study of Re-Biopsy in EGFR-TKI- Resistant Patients with EGFR Mutation-Positive Advanced NSCLC
}

Kenichi Koyama

Niigata Cancer Center Hospital

\section{Satoru Miura}

Niigata Cancer Center Hospital

Satoshi Watanabe ( $\sim$ satoshi7@med.niigata )

Niigata University

Satoshi Shoji

Niigata University

Jun Koshio

Nagaoka Red Cross Hospital

Yoshiki Hayashi

Nagaoka Chuo General Hospital

Daisuke Ishikawa

Niigata Prefectural Central Hospital

Ko Sato

Niigata Prefectural Central Hospital

Takao Miyabayashi

Niigata City General Hospital

Masaaki Okajima

Saiseikai Niigata Hospital

\section{Takeshi Ota}

Niigata Prefectural Shibata Hospital

Tomohiro Tanaka

Niigata University

Naoya Matsumoto

Nishi Niigata Chuo National Hospital

Hideyuki Kuriyama

Niigata Medical Center

Tetsuya Abe

Niigata City General Hospital

Koichiro Nozaki 
Niigata University

\section{Kosuke Ichikawa}

Saiseikai Niigata Hospital

\section{Rie Kondo}

Niigata University

Hiroshi Tanaka

Niigata Cancer Center Hospital

\section{Toshiaki Kikuchi}

Niigata University

\section{Research Article}

Keywords: Non-small cell lung cancer, Epidermal growth factor receptor, re-biopsy, osimertinib

Posted Date: December 8th, 2021

DOl: https://doi.org/10.21203/rs.3.rs-1088650/v1

License: (c) (i) This work is licensed under a Creative Commons Attribution 4.0 International License. Read Full License 
Original Article

Observational Study of Re-Biopsy in EGFR-TKI-Resistant Patients with EGFR Mutation-Positive Advanced NSCLC.

Authors

Kenichi Koyama ${ }^{1}$, Satoru Miura ${ }^{1}$, Satoshi Watanabe ${ }^{2}$, Satoshi Shoji ${ }^{2}$, Jun Koshio ${ }^{3}$, Yoshiki Hayashi ${ }^{4}$, Daisuke Ishikawa ${ }^{5}$, Ko Sato ${ }^{5,}$, Takao Miyabayashi ${ }^{6}$, Masaaki Okajima $^{7}$, Takeshi Ota ${ }^{8}$, Tomohiro Tanaka ${ }^{2}$, Naoya Matsumoto ${ }^{9}$, Hideyuki Kuriyama ${ }^{13}$, Tetsuya Abe ${ }^{6,12}$, Koichiro Nozaki ${ }^{2}$, Kosuke Ichikawa ${ }^{7}$, Rie Kondo ${ }^{2}$, Hiroshi Tanaka ${ }^{1}$, Toshiaki Kikuchi ${ }^{2}$

Affiliations

1. Department of Internal Medicine, Niigata Cancer Center Hospital

2. Department of Respiratory Medicine and Infectious Diseases, Niigata University Graduate School of Medical and Dental Sciences

3. Department of Respiratory Medicine, Nagaoka Red Cross Hospital

4. Department of Respiratory Medicine, Nagaoka Chuo General Hospital

5. Department of Respiratory Medicine, Niigata Prefectural Central Hospital

6. Department of Respiratory Medicine, Niigata City General Hospital

7. Department of Respiratory Medicine, Saiseikai Niigata Hospital

8. Department of Respiratory Medicine, Shibata Hospital - Niigata Prefectural Hospital

9. Department of Respiratory Medicine, Nishi Niigata Chuo Hospital 
10. Department of Respiratory Medicine, Sado General Hospital

11. Department of Respiratory Medicine, Tsuruoka Municipal Shonai Hospital

12. Department of Respiratory Medicine, Shinrakuen Hospital

13.Department of Respiratory Medicine, Niigata Medical Center

Correspondence

Satoru Miura, M.D., Ph.D.

Department of Internal Medicine, Niigata Cancer Center Hospital

2-15-3, Kawagishi-cho, Chuo-ku, Niigata city, 951-8566, Japan

Tel.: +81-25-266-5111, Fax: +81-25-233-3849

E-mail:_miusat1118@niigata-cc.jp

Satoshi Watanabe, M.D., Ph.D.

Department of Respiratory Medicine and Infectious Diseases, Niigata University

Graduate School of Medical and Dental Sciences, 1-757 Asahimachidori, Chuouku, Niigata, 951-8510, Japan. TEL: (81)25-368-9324. FAX: (81)25-368-9326.

Email: satoshi7@med.niigata-u.ac.jp.

Keywords

Non-small cell lung cancer, Epidermal growth factor receptor, re-biopsy, osimertinib

Word count: 4109

Number of Figures: 1

Number of Tables: 3 


\begin{abstract}
Identification of acquired resistant mutation has been essential in non-small cell lung cancer (NSCLC) patients with epidermal growth factor receptor (EGFR) active mutations. Re-biopsy plays a pivotal role to select the optimal treatment for patients who develop resistance to initial EGFR-tyrosine kinase inhibitors (EGFR-TKI). This multicenter, observational study was conducted to investigate the details of re-biopsy in Japanese clinical practice. The primary endpoints were the implementation rate of re-biopsy and the concordance rate for the T790M mutation detection between histology and cytology specimens using the Cobas ${ }^{\circledR} E G F R$ mutation test v2.194 patients with EGFR-mutant NSCLC were enrolled and 120 patients developed acquired resistance to EGFR-TKIs. The median age was 68 years (range $20-87$ ), and $52.5 \%$ of the patients were women. Rebiopsy was performed on 109 patients with the implementation rate of re-biopsy was $90.8 \%$. The success rate of re-biopsy in total/histology/cytology/liquid biopsy population was $78.0 \%, 94.9 \%, 83.3 \%$ and $43.8 \%$, respectively. The positive percent agreement and the negative percent agreement in the detection of T790M mutations between the histology and cytology specimens was both of $90.9 \%$.

Aggressive obtaining histological or cytological tissue samples at re-biopsy may contribute to improvement of the detection rate of T790M mutation. (trial registration number: UMIN000026019)
\end{abstract}




\section{Introduction}

Molecular targeted therapies and immunotherapy using immune checkpoint inhibitors have emerged as the essential modalities in various cancer treatments. Epidermal growth factor receptor (EGFR) mutations were strongly correlated with the clinical benefit of EGFR-tyrosine kinase inhibitors (EGFR-TKIs), and the first druggable driver mutation in non-small cell lung cancer (NSCLC) ${ }^{1,2}$. It has been reported that the firstor second-generation EGFR-TKIs showed significant clinical benefits compared with the standard chemotherapy in patients with EGFR active mutations ${ }^{3-8}$. After the discovery of $E G F R$ active mutations, various of druggable driver mutations and tyrosine kinase inhibitors were identified in lung adenocarcinoma, such as EML4-ALK, ROS1, and $B R A F$ mutations. The tyrosine kinase inhibitors for these kinds of druggable driver mutations were highly recommended in the current treatment guidelines for NSCLC ${ }^{9-11}$. In current clinical practice, the detection of druggable somatic gene alterations has been essential to select the adequate treatments in the treatment of NSCLC ${ }^{12}$.

NSCLC patients with EGFR active mutations respond to EGFR-TKI for a certain period of time, but most of them will have disease progression with acquired resistance after a median of 12 months. The mechanisms of acquired resistance have been identified. The most prevalent resistance mutation was the Thr790Met (T790M) point mutation at exon 20 of which revealed in approximately half of the first- or second-generation EGFRTKI resistant patients. Osimertinib is a third-generation, irreversible EGFR-TKI that selectively inhibits not only EGFR active mutation but also T790M. The AURA 3 trial was a phase 3 trial to elucidate the efficacy of osimertinib compared with platinum doublet chemotherapy in patients with T790M positive advanced NSCLC, who had disease progression after first-line EGFR-TKI ${ }^{13}$. This trial demonstrated that osimertinib 
have significantly greater clinical effect than chemotherapy for T790M positive NSCLC patients. Based on this result, osimertinib was approved for patients who were identified the T790M mutations. The analysis of the T790M mutations needs to obtain the histological specimens from the relapsing sites using "re-biopsy". However, there are some issues to perform the re-biopsy, such as difficulty access the relapsing site, patient rejection because of invasive procedures ${ }^{14}$. Now, the liquid biopsy method to identify the T790M mutation has been available to resolve these issues. However, the detection sensitivity of the T790M mutation using liquid biopsy is relatively low, and it is difficult to apply the liquid biopsy methods to all of resistant patients. In addition, there is limited data on the appropriateness of cytological specimens to test the T790M mutations using the Cobas ${ }^{\circledR}$ EGFR Mutation Test v2 if a suitable tissue specimen was not obtained. Therefore, we conducted this multicenter, prospective observational study to investigate the details of re-biopsy including the implementation or successful rate of re-biopsy and the concordance rate for the T790M mutation detection between histological and cytological specimens in EGFR-TKI resistant NSCLC patients. 


\section{Results}

\section{Patient characteristics in patients with acquired resistance}

A total of 194 patients was enrolled from February 2017 to January 2019. Among these, 120 patients were defined as acquired resistance to ongoing EGFR-TKI therapy and considered performing re-biopsy (Fig. 1). Characteristics of patients who received rebiopsy are shown in Table 1. The reasons for acquired resistance were as follows: One hundred-four patients (86.7\%) were RECIST-PD, 60 were clinical PD, and 8 were gradual progressive SD. The median age was 68 years (range, 20-87), and $52.5 \%$ of the patients were women. Adenocarcinoma (99.2\%) was the most common histology. Sixty-six patients (55.0\%) had exon 19 deletions, 45 (37.5\%) had L858R point mutations, and 10 (8.4\%) had uncommon mutations such as G719X, L861Q and compound mutation. Fortyseven patients (39.2\%) had been treated with erlotinib, 36 patients $(30 \%)$ had been treated with gefitinib, and 37 patients $(30.3 \%)$ had been treated with afatinib at disease progression. Median treatment duration of the start of EGFR-TKIs to re-biopsy was 14 months (range, 1.9-90 months).

\section{Results of re-biopsy}

Re-biopsy was performed on 109 patients $(90.8 \%)$, while 11 patients $(9.2 \%)$ did not receive re-biopsy (Fig. 1). The implementation rate of re-biopsy was $90.8 \%$. Reasons for not performing re-biopsy were as follows: difficult to approach to relapse sites $(n=5)$, patient refusal $(n=2)$, transfer to a different hospital $(n=3)$ and previously performed re-biopsy $(\mathrm{n}=1)$.

Tissue histology specimens were obtained from 59 patients (54.1\%) including: 27 (45.8\%) transbronchial biopsy using endobronchial ultrasonography with a guide-sheath 
(EBUS-GS); 8 (13.6\%) ultrasound-guided core needle liver biopsy; 6 (10.2\%) transbronchial biopsy without EBUS-GS; 5 (8.5\%) endobronchial ultrasonographyguided transbronchial needle aspiration (EBUS-TBNA); 5 (8.5\%) video-assisted thoracic surgery (VATS); 4 (6.8\%) superficial lymph node biopsy; 3 (5.1\%) bone biopsy using computed tomography (CT)-guided percutaneous core needle biopsy (PCNB); 1 (1.8\%) lung biopsy using CT-guided PCNB. For 18 patients (16.5\%), cytology specimens were only available including: 13 (72.2\%) thoracentesis; 3 (18.8\%) lumber puncture; 1 (5.6\%) bronchoscopy brushing;1 (5.6\%) superficial lymph node biopsy.

The summary of re-biopsy results is shown in Table 2. In 59 patients, tissue histological specimens were diagnosed as follows: adenocarcinoma $(n=53,90 \%)$, transformation to other histology $(n=3,5.1 \%$. Two were small cell lung carcinoma and 1 was sarcomatoid cancer). The success rate of re-biopsy using histological procedures was a $94.9 \%(56 / 59)$. In adenocarcinoma patients, the T790M mutations were identified in $32(54.2 \%)$ patients. In cytology specimens obtained from 18 patients, malignant cells revealed in 15 cytology specimens (83.3\%). From cytology specimens, the T790M mutations were identified in only 1 patient (5.6\%). The success rate of re-biopsy using cytological procedures was an $83.3 \%(15 / 18)$. In 32 patients receiving re-biopsy by the plasma liquid biopsy, the T790M mutations were identified in 8 patients $(25 \%)$ and negative for T790M mutations in 6 patients $(18.8 \%)$. Eighteen patients $(56.2 \%)$ could not have any information of T790M mutations from liquid biopsy due to poor PCR products. Thus, the success rate of liquid biopsy was $43.8 \%$ (14/32). In total, the T790M mutations were identified in 41 patients $(37.6 \%)$ out of 109 patients who received initial re-biopsy. The total success rate of re-biopsy was 78.0\% (85/109). Additional re-biopsy after negative results of initial re-biopsy was performed in 7 patients. Among the 7 
patients who underwent the second re-biopsy, T790M mutations were revealed in 2 patients $(28.6 \%)$ by the cytology procedures. Finally, the T790M mutations were identified in 43patients $(43 / 109,39.4 \%)$ in this observational cohort including first and second re-biopsy. The problematic complications regarding re-biopsy were not reported.

\section{The concordance rate for the T790M mutation detection between histology and cytology specimens}

There were 22 patients who had both the histology and cytology specimens obtained at the same time for the T790M mutation testing (Table 3). The detection rate of the T790M mutations in the histology or cytology specimens was both the same at $90.9 \%$ (10/11). Each group had one patient who was discrepancy for T790M mutation result. As a result, the positive percent agreement and the negative percent agreement was both of $90.1 \%$. 


\section{Discussion}

The importance of re-biopsy has increasing in patients with EGFR mutation after the report of the AURA3 study which demonstrated the efficacy of osimertinib for patients with T790M positive NSCLC patients. It is suggested that the implementation rate of rebiopsy may be associated with the prognosis of EGFR-TKI refractory NSCLC patients ${ }^{15}$. In the current observational study, the implementation rate of re-biopsy was $93.4 \%$ with the T790M positive rate was $37.6 \%$ in the first re-biopsy. In our knowledge, the implementation rate of re-biopsy was highest compared with the previous retrospective or observational studies regarding the T790M detection. The implementation rate of rebiopsy has been reported as ranged $55.1-63.0 \%$ in the retrospective studies or $86.9 \%$ in the Japanese observational study named the REMEDY study ${ }^{14,16,17}$. The use of liquid biopsy obviously might affect the high implementation rate of re-biopsy. The liquid biopsy has significant advantages of feasibility, such as non-invasiveness and accessibility, to detecting the T790M mutations ${ }^{18}$. Actually, the most common procedure of re-biopsy in the REMEDY study was the liquid biopsy using plasma, accounting for a $58.1 \%{ }^{16}$. In the current study, $28.9 \%$ of patients received the liquid biopsy as re-biopsy procedure. When this study was conducted, the liquid biopsy was not approved in clinical practice in Japan. The main limitation of this study was that the implementation rate of re-biopsy could not be evaluated according to the original statistical hypothesis because of the approval of liquid biopsy in Japan. However, the liquid biopsy for EGFR-TKI resistant patients have some limitation. First problem is the transformation to other histopathological features, such as small cell lung carcinoma (SCLC) transformation, cannot detect by liquid biopsy ${ }^{19}$. There were four transformation cases ( 3 small cell lung carcinoma and one sarcomatoid cancer) in this 
study. The efficacy of osimertinib may be limited for the concomitant case of the T790M mutation and SCLC transformation ${ }^{20}$. Second problem of liquid biopsy using plasma is the lower detection rate of the T790M mutation. Previous study demonstrated that one third of patients who performed liquid biopsy revealed the T790M mutations with the Cobas ${ }^{\circledR}$ EGFR Mutation Test ${ }^{21}$. In this study, the success rate of liquid biopsy was $43.8 \%$ and the detection rate of the T790M mutation was $25 \%$. These results indicated that liquid biopsy could be a useful option for patients whom tissue samples cannot be obtained.

The successful re-biopsy was important to determine the subsequent treatments for EGFR-TKI refractory patients. In this study, the success rate of histological procedure (94.9\%) was higher compared with cytological procedure $(83.3 \%)$ or liquid biopsy (43.8\%). The main reason for the selection of liquid biopsy as a re-biopsy procedure was the inaccessibility to relapse lesions, such as bone metastasis, central nerves metastasis or small lung metastases (data not shown). The skillful expert and adequate devices will be needed to perform the invasive procedures accessing such relapse lesions, and it may depend on the status of participating institutions. It is important to cooperate with each institution in local communities to improve the success rate of rebiopsy.

The concordance of tissue or plasma samples was well investigated in previous report ${ }^{22}$. However, the data regarding the concordance of histological and cytological samples has been insufficient. The transbronchial procedures has been the common methods to perform re-biopsy because of availability and feasibility of bronchoscopy. The percentage of patients who performed re-biopsy transbronchial procedures without liquid biopsy was 50.6\% (39/77) including EBUS-GS, transbronchial biopsy, EBUS- 
TBNA and BF brushing in this study (Fig. 1). The percentage of re-biopsy procedure transbronchial procedures in prospective cohort trials was $52.4 \%(43 / 82)$ and $63.9 \%$ $(69 / 108)^{16,23}$. However, it is difficult to obtain the sufficient tissue samples using transbronchial procedures from all of the patients. We investigated the accuracy of cytology-based T790M detection compared with histology-based testing using the tissues obtained at the same time. As a result, the positive percent agreement and the negative percent agreement was both of $90.1 \%$ (Table 3 ). This result indicated that cytology specimens obtained by transbronchial procedures may be useful to detecting the T790M mutations.

Revealing the resistant mechanisms and developing strategy of overcoming the resistance of EGFR-TKIs remains main focuses of the investigation of EGFR mutation positive patients. After the report of FLAURA study, the importance of T790M detection has decreased because most of physicians tend to choose osimertinib for chemo-naive EGFR mutation positive NSCLC patients. However, the new resistant mechanisms have been identified from the analysis using liquid biopsy after treatment of osimertinib in the FLAURA trial ${ }^{24}$. The most frequent resistance mechanisms were MET amplification (15\%) and secondary EGFR mutations (10\%) including C797X and uncommon mutations. These resistant mechanisms will become new targets to overcome the resistance of osimertinib, and a number of clinical trials have been ongoing in this area. The clinical investigations concerning re-biopsy procedures and mutation detecting methods should be continued for future driver-based medicine. The current study demonstrated that the re-biopsy was feasible and could provide the useful data to select the subsequent treatments even in clinical practice. 


\section{Methods}

\section{Study design}

The present study was a multicenter, prospective, observational study in patients with EGFR mutation-positive advanced NSCLC who have experienced disease progression during treatment with EGFR-TKI. Patients with advanced NSCLC meeting all inclusion criteria and not meeting any exclusion criteria were consecutively enrolled from medical institutions participating in the Niigata Lung Cancer Treatment Group (NLCTG) to collect information on re-biopsy data. This study was conducted according to the principles of the Declaration of Helsinki. The Institutional Review Board of each participating institution (Niigata Cancer Center Hospital, Niigata University Graduate School of Medical and Dental Sciences, Nagaoka Red Cross Hospital, Nagaoka Chuo General Hospital, Niigata Prefectural Central Hospital, Niigata City General Hospital, Saiseikai Niigata Hospital, Shibata Hospital - Niigata Prefectural Hospital, Nishi Niigata Chuo Hospital, Sado General Hospital, Tsuruoka Municipal Shonai Hospital, Shinrakuen Hospital, Niigata Medical Center)approved the protocol. This study is registered at February 2017 on the clinical trials site of the University Hospital Medical Information Network Clinical Trials Registry (registration number: UMIN000026019). The information on patient demographics was collected retrospectively from the medical records at the study enrollment. Additional data regarding re-biopsy was collected when following situations; sustained response to EGFR-TKI (compete response, pertain response, or stable disease more than 6 weeks) and acquired resistance to ongoing EGFR-TKI. The definition of acquired resistance in this study was as follows: progressive disease according to Response Evaluation Criteria in Solid Tumors (RECIST) criteria (RECIST-PD), clinically progressive disease assessed by physicians 
(clinical PD), and stable disease according to RECIST criteria but increasing tumor volume compared with baseline (gradual progressive-SD). The collected data was as follows: patient status at the point of re-biopsy, the information of re-biopsy sites (relapse site, size, number, and so on) and procedure of re-biopsy, results of re-biopsy (cytology, histology, and the T790M status), complications regarding re-biopsy, subsequent treatments, and prognosis. If patients were not performed re-biopsy at acquired resistance, the reasons of not undergoing re-biopsy including patient's refusal were collected. For patients with both of histological and cytological specimens available, the concordance rate for the T790M mutation detection between both specimens were analyzed by the Cobas ${ }^{\circledR} E G F R$ Mutation Test v2. All data was collected using the electronic data capture.

\section{Patients}

The eligibility criteria were as follows: a histologically or cytologically confirmed advanced NSCLC with EGFR active mutations; stage III/IV not amenable to definitive radiation therapy or postoperative recurrence at the start of EGFR-TKI therapy; ongoing EGFR-TKI therapy (gefitinib, erlotinib or afatinib); no prior T790M mutation detection; written informed consent for study participation from the patient. Patients who treated with third generation EGFR-TKI was excluded. Written informed consent was obtained from all the study participants

\section{Study endpoints}

The primary endpoint was the implementation rate of re-biopsy defined as the number of patients performed re-biopsy from any sites/total number of enrolled patients. After approval of liquid biopsy testing for T790M mutation on July 2017, liquid biopsy was also counted as re-biopsy. The co-primary endpoint was the concordance rate for the 
T790M mutation detection between histology and cytology specimens using the Cobas ${ }^{\circledR}$ EGFR mutation test v2. Cell block analysis was included in cytology specimens. Secondary endpoints were as follows; the success rate of re-biopsy according to procedure, the T790M mutation positive rate according to biopsy site or procedure, safety of re-biopsy procedures, reasons for patients not undergoing re-biopsy, implementation rate of re-biopsy according to institution and available re-biopsy procedures. The success rate of re-biopsy was defined as the percentage of cases in which malignant cells and the result of gene mutation was available among the cases who underwent re-biopsy. The patients revealed the transformation to other histologies were included in the re-biopsy successful cases.

\section{Statistical analyses}

In the retrospective study regarding re-biopsy, the implementation rate of re-biopsy was reported as $62.5 \%$, and the percentage of patients who could examine the EGFR gene mutation using histological specimens was reported as $28 \%{ }^{14}$. We estimated the lower limit to be able to examine the T790M mutations in both of histology and the cytology specimens was $30 \%$. If we assumed threshold rate of 0.5 , expected rate of $0.8,83$ patients will be needed to evaluate the positive percent agreement of T790M detection between histology and cytology specimens with $80 \%$ power, and alpha level of 0.05 (2sided). Even if the dropout of about $5 \%$ is considered, it is estimated that 100 patients is a target number of patients who had acquired resistance. This sample size would have half-width of exact $95 \%$ confidence interval of $+/-10 \%$ or less for the implementation rate of re-biopsy. The SAS version 9.4 (SAS Inc., Cary, NC, USA) were used for the sample size evaluation. 


\section{Acknowledgments}

We thank the patients and their families and Hiroko Aita for their support and participation in this study.

This study was funded by AstraZeneca Japan.

\section{Declarations}

Satoshi Watanabe received honoraria (lecture fee) from AstraZeneca, CHUGAI

PHARMACEUTICAL, ONO PHARMACEUTICAL , Eli Lilly Japan, Bristol-Myers, Boehringer Ingelheim, MSD, TAIHO PHARMACEUTICAL, Pfizer, Novartis, DAIICHI SANKYO. Satoshi Watanabe received research grants from AstraZeneca, Boehringer Ingelheim. Hiroshi Tanaka received honoraria (lecture fee) from AstraZeneca. Toshiaki Kikuchi received honorarium (lecture fee) from Boehringer Ingelheim, AstraZeneca, CHUGAI PHARMACEUTICAL .

Toshiaki Kikuchi received research fund from AstraZeneca.

Toshiaki Kikuchi received research grants from SHIONOGI PHARMACEUTICAL, CHUGAI PHARMACEUTICAL, ONO PHARMACEUTICAL, Eli Lilly Japan. Other authors did not have any competing interest. 


\section{Reference}

1. Lynch TJ, Bell DW, Sordella R, et al. Activating mutations in the epidermal growth factor receptor underlying responsiveness of non-small-cell lung cancer to gefitinib. The New England journal of medicine 2004;350:2129-39.

2. Paez JG, Janne PA, Lee JC, et al. EGFR mutations in lung cancer: correlation with clinical response to gefitinib therapy. Science (New York, NY) 2004;304:1497-500.

3. Maemondo M, Inoue A, Kobayashi K, et al. Gefitinib or chemotherapy for non-small-cell lung cancer with mutated EGFR. The New England journal of medicine 2010;362:2380-8.

4. Mitsudomi T, Yatabe Y. Mutations of the epidermal growth factor receptor gene and related genes as determinants of epidermal growth factor receptor tyrosine kinase inhibitors sensitivity in lung cancer. Cancer science 2007;98:1817-24.

5. Rosell R, Carcereny E, Gervais R, et al. Erlotinib versus standard chemotherapy as first-line treatment for European patients with advanced EGFR mutation-positive non-small-cell lung cancer (EURTAC): a multicentre, open-label, randomised phase 3 trial. The Lancet Oncology 2012;13:239-46.

6. Zhou C, Wu YL, Chen G, et al. Erlotinib versus chemotherapy as first-line treatment for patients with advanced EGFR mutation-positive non-small-cell lung cancer (OPTIMAL, CTONG-0802): a multicentre, open-label, randomised, phase 3 study. The Lancet Oncology 2011;12:735-42.

7. Sequist LV, Yang JC, Yamamoto N, et al. Phase III study of afatinib or cisplatin plus pemetrexed in patients with metastatic lung adenocarcinoma with EGFR mutations. J Clin Oncol 2013;31:3327-34.

8. $\quad \mathrm{Wu} \mathrm{YL}, \mathrm{Zhou} \mathrm{C,} \mathrm{Hu} \mathrm{CP,} \mathrm{et} \mathrm{al.} \mathrm{Afatinib} \mathrm{versus} \mathrm{cisplatin} \mathrm{plus} \mathrm{gemcitabine} \mathrm{for} \mathrm{first-line} \mathrm{treatment}$ of Asian patients with advanced non-small-cell lung cancer harbouring EGFR mutations (LUX-Lung 6): an open-label, randomised phase 3 trial. The Lancet Oncology 2014;15:213-22.

9. Hanna N, Johnson D, Temin S, et al. Systemic Therapy for Stage IV Non-Small-Cell Lung Cancer: American Society of Clinical Oncology Clinical Practice Guideline Update. J Clin Oncol 2017;35:3484-515.

10. Planchard D, Popat S, Kerr K, et al. Metastatic non-small cell lung cancer: ESMO Clinical Practice Guidelines for diagnosis, treatment and follow-up. Ann Oncol 2018;29:iv192-iv237.

11. Akamatsu H, Ninomiya K, Kenmotsu H, et al. The Japanese Lung Cancer Society Guideline for non-small cell lung cancer, stage IV. Int J Clin Oncol 2019;24:731-70.

12. Kalemkerian GP, Narula N, Kennedy EB, et al. Molecular Testing Guideline for the Selection of Patients With Lung Cancer for Treatment With Targeted Tyrosine Kinase Inhibitors: American Society of Clinical Oncology Endorsement of the College of American Pathologists/International Association for the Study of Lung Cancer/Association for Molecular Pathology Clinical Practice Guideline Update. J Clin Oncol 2018;36:911-9.

13. Mok TS, Wu YL, Ahn MJ, et al. Osimertinib or Platinum-Pemetrexed in EGFR T790M-Positive Lung Cancer. The New England journal of medicine 2017;376:629-40. 
14. Kawamura T, Kenmotsu H, Omori S, et al. Clinical Factors Predicting Detection of T790M Mutation in Rebiopsy for EGFR-Mutant Non-small-cell Lung Cancer. Clin Lung Cancer 2018;19:e247e52.

15. Imakita T, Matsumoto H, Hirano K, Morisawa T, Sakurai A, Kataoka Y. Impact on prognosis of rebiopsy in advanced non-small cell lung cancer patients after epidermal growth factor receptor-tyrosine kinase inhibitor treatment: a systematic review. BMC Cancer 2019;19:105.

16. Seto T, Nogami N, Yamamoto N, et al. Real-World EGFR T790M Testing in Advanced NonSmall-Cell Lung Cancer: A Prospective Observational Study in Japan. Oncol Ther 2018;6:203-15.

17. Tseng JS, Su KY, Yang TY, et al. The emergence of T790M mutation in EGFR-mutant lung adenocarcinoma patients having a history of acquired resistance to EGFR-TKI: focus on rebiopsy timing and long-term existence of T790M. Oncotarget 2016;7:48059-69.

18. Li C, Jia R, Liu H, Zhang B, Wang C. EGFR T790M detection and osimertinib treatment response evaluation by liquid biopsy in lung adenocarcinoma patients with acquired resistance to first generation EGFR tyrosine kinase inhibitors. Diagn Pathol 2018;13:49.

19. Oser MG, Niederst MJ, Sequist LV, Engelman JA. Transformation from non-small-cell lung cancer to small-cell lung cancer: molecular drivers and cells of origin. The Lancet Oncology 2015;16:e16572.

20. Minari R, Bordi $\mathrm{P}$, Del Re $\mathrm{M}$, et al. Primary resistance to osimertinib due to SCLC transformation: Issue of T790M determination on liquid re-biopsy. Lung Cancer 2018;115:21-7.

21. Thress KS, Brant R, Carr TH, et al. EGFR mutation detection in ctDNA from NSCLC patient plasma: A cross-platform comparison of leading technologies to support the clinical development of AZD9291. Lung Cancer 2015;90:509-15.

22. Zhou J, Zhao C, Zhao J, et al. Re-biopsy and liquid biopsy for patients with non-small cell lung cancer after EGFR-tyrosine kinase inhibitor failure. Thorac Cancer 2019;10:957-65.

23. Chouaid C, Dujon C, Do P, et al. Feasibility and clinical impact of re-biopsy in advanced non small-cell lung cancer: a prospective multicenter study in a real-world setting (GFPC study 12-01). Lung Cancer 2014;86:170-3.

24. Ramalingam SS, Cheng Y, Zhou C, et al. Mechanisms of acquired resistance to first-line osimertinib: Preliminary data from the phase III FLAURA study. Ann Oncol 2018;29 Suppl 8:viii740. 
Figure legend

Figure 1: Overview of patient status for enrolled patients.

One hundred twenty patients were defined as acquired resistance to EGFR-TKIs and 109 patients underwent re-biopsy. 


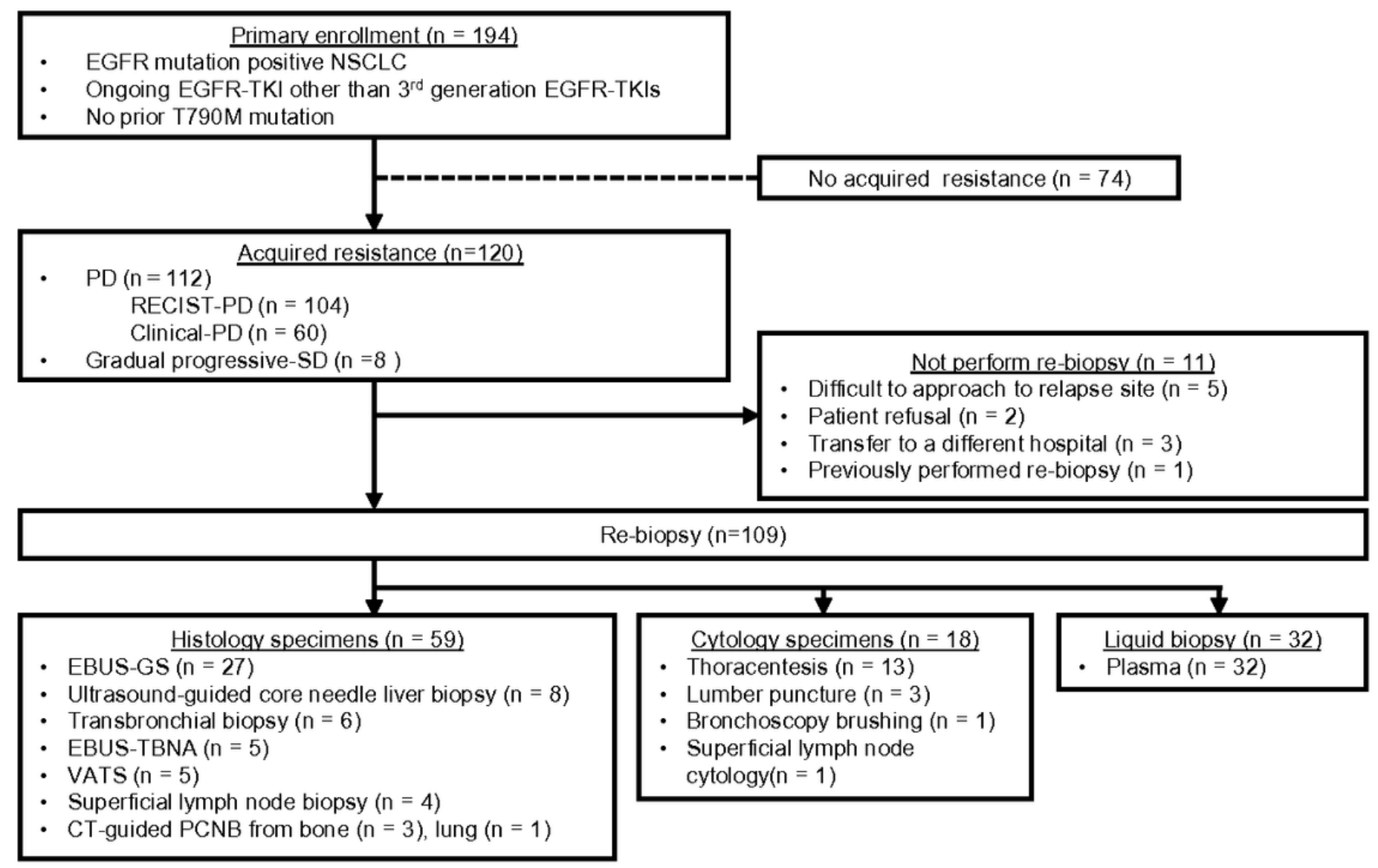

Figure 1

Overview of patient status for enrolled patients. One hundred twenty patients were defined as acquired resistance to EGFR-TKIs and patients underwent re-biopsy.

\section{Supplementary Files}

This is a list of supplementary files associated with this preprint. Click to download.

- NLCTG1602Table1.pdf

- NLCTG1602Table2.pdf

- NLCTG1602Table3.pdf 